\title{
Creation of a Decision Support Tool for Expectant Parents Facing Threatened Periviable Delivery: Application of a User-Centered Design Approach
}

\author{
Brownsyne Tucker Edmonds ${ }^{1} \cdot$ Shelley M. Hoffman ${ }^{1} \cdot$ Dustin Lynch $^{2} \cdot$ Erin Jeffries $^{1} \cdot$ Kelli Jenkins $^{2} \cdot$ Sarah Wiehe $^{2}$. \\ Nerissa Bauer ${ }^{2} \cdot$ Miriam Kuppermann $^{3}$
}

Published online: 29 November 2018

(c) Springer Nature Switzerland AG 2018

\begin{abstract}
Background Shared decision-making (SDM) is optimal in the context of periviable delivery, where the decision to pursue life-support measures or palliation is both preference sensitive and value laden. We sought to develop a decision support tool (DST) prototype to facilitate SDM by utilizing a user-centered design research approach.

Methods We convened four patient and provider advisory boards with women and their partners who had experienced a surviving or non-surviving periviable delivery, pregnant women who had not experienced a prior preterm birth, and obstetric providers. Each 2-h session involved design research activities to generate ideas and facilitate sharing of values, goals, and attitudes. Participant feedback shaped the design of three prototypes (a tablet application, family story videos, and a virtual reality experience) to be tested in a final session.

Results Ninety-five individuals (48 mothers/partners; 47 providers) from two hospitals participated. Most participants agreed that the prototypes should include factual, unbiased outcomes and probabilities. Mothers and support partners also desired comprehensive explanations of delivery and care options, while providers wanted a tool to ease communication, help elicit values, and share patient experiences. Participants ultimately favored the tablet application and suggested that it include family testimonial videos.

Conclusion Our results suggest that a DST that combines unbiased information and understandable outcomes with family testimonials would be meaningful for periviable SDM. User-centered design was found to be a useful method for creating a DST prototype that may lead to improved effectiveness, usability, uptake, and dissemination in the future, by leveraging the expertise of a wide range of stakeholders.
\end{abstract}

This study was presented as an oral presentation at the 9th Annual International Shared Decision Making (ISDM) conference in Lyon, France in July 2017.

Electronic supplementary material The online version of this article (https://doi.org/10.1007/s40271-018-0348-y) contains supplementary material, which is available to authorized users.

Brownsyne Tucker Edmonds

btuckere@iupui.edu

1 Department of Obstetrics and Gynecology, Indiana University School of Medicine, 410 W. 10th Street, Indianapolis, IN 46202, USA

2 Patient Engagement Core, Community Health Partnerships, Indiana Clinical and Translational Sciences Institute, Indiana University School of Medicine, 410 W. 10th Street, Indianapolis, IN 46202, USA

3 Department of Obstetrics, Gynecology and Reproductive Sciences, University of California, 550 16th St, Box 0132, San Francisco, CA 94143, USA

\section{Key Points for Decision Makers}

We created a new decision support tool to improve the quality of periviable counseling, facilitate patient-centered care, and enhance patient-provider communication by providing outcome estimates for neonatal survival and disability and incorporating values clarification into the decision support process.

We utilized a novel application of user-centered design to engage key stakeholders in every aspect of tool development and design. 


\section{Introduction}

Periviable neonates, delivered between 20 and 26 weeks gestational age [1] bear the greatest burden of infant mortality and morbidity. Born too early to survive outside of the womb without ventilator support and intensive care, roughly half of these neonates will die, and among survivors, more than two-thirds will suffer moderate to severe cognitive and physical impairments [2-5]. Despite advances in neonatal intensive care, long-term neurodevelopmental outcomes for these infants have improved by only modest amounts [6, 7]. These births are both financially and emotionally costly [8-10]; the Centers for Disease Control has estimated that extreme prematurity results in US \$26 billion in annual healthcare costs [11], and parents' long-term mental health and functioning can be compromised, resulting in depression, anxiety, and post-traumatic stress disorder [12].

Decision-making regarding periviable resuscitation presents prospective parents and physicians with the unfortunate challenge of making 'end-of-life' decisions at the very beginning of life. Because survival is relatively infrequent and outcomes are poor, the American Academy of Pediatrics calls for shared decision-making (SDM) when families face resuscitation decisions, so that parents are engaged in deliberations, and, ultimately, choose whether to attempt resuscitation or pursue palliation $[13,14]$. Though parents may have religious or cultural values that inform their preferences [15], it is unlikely that they have considered this decision as a possibility until they are faced with it. As a result, when facing this situation, they may initially have limited insight into the attitudes, values, or goals of care that shape their preferences.

Despite the American Academy of Pediatrics' recommendations, along with patient desires for SDM [14, 16-18], the current model of periviable decision-making is not shared, well informed, or patient centered [19-23]. In previous work, we found that physicians are not skilled at eliciting values and preferences, or in helping parents to clarify their priorities in the course of periviable counseling. Though they frequently acknowledge that the decision depends on the parents' values, physicians do not typically assist parents in the deliberation needed for preference construction [22, 23]. In addition, mortality and morbidity estimates that are provided are variable and inaccurate [16]; there is conflicting guidance regarding antenatal interventions, such as steroid administration; and goals of care or resuscitation preferences are not always elicited [21]. By presenting parents with inconsistent information without eliciting and clarifying values and goals of care, providers place parents at substantial risk for misinformed decision-making, decisional conflict, and regret, which may have long-term implications for parental mental health and quality of life.
Decision support tools (DSTs) are used to assist providers in the task of conveying complex information to patients and facilitating values clarification in service of creating more shared, informed decision-making encounters for patients and providers. User-centered design (UCD) has the promise to ensure that DSTs are designed with end-users-in our case, providers, patients and their family members-in mind. These approaches to design are 'human centered', in that the end result of the design process is intended to be intuitive, and that the process by which the products, systems, and services are designed is collaborative, with stakeholders included as partners at every step of the design process. In an effort to facilitate more patient-centered periviable counseling encounters, we set out to utilize a UCD approach in developing a prototype of a DST, which we call the Periviable GOALS (Getting Optimal Alignment around Life Support decisions) tool, to facilitate informed SDM regarding whether to opt for neonatal resuscitation versus comfort care in the context of periviable delivery. Our primary objectives were to (1) engage patient and provider advisory boards (PABs), to identify attitudes, values, and goals of care that drive resuscitation decisions; and (2) use generative data from these PABs to co-design a decision support GOALS tool prototype suitable to test in a future, planned, multisite, randomized controlled trial.

\section{Methods}

This study was conducted by Indiana University (IU) Department of Obstetrics and Gynecology in partnership with the Indiana Clinical and Translational Sciences Institute Patient Engagement Core and the University of California at San Francisco (UCSF). The institutional review boards at IU (IRB protocol \#1606279321) and UCSF (IRB protocol \#16-20705) approved this study.

\subsection{User-Centered Design Research}

The term 'user-centered design' (UCD) has its roots in human-computer interaction, industrial design, and cognitive psychology. This approach incorporates information about the people who will use the product and studies how they interact with it to develop intuitive tools and systems for people to use [24, 25]. These approaches to design are 'human centered', in that the end result of the design process is intended to be intuitive, and that the process by which the products, systems, and services are designed is collaborative-with stakeholders included as partners at every step of the design process [26]. While UCD sessions have some similarities to traditional focus groups, in that it is a "research technique that collects data through group interaction on a topic determined by the researcher" [27], 
UCD techniques differ in important ways from conventional focus group testing. UCD does not typically rely on simply asking questions in a group interview style typical of focus groups. The kind of explicit knowledge that is gained by asking questions in this manner is often already known. Instead, in UCD sessions, participants participate in a series of generative activities. One author explains, "People can't always tell you in words about their unmet needs. If they could, they would probably no longer be unmet" [28]. To overcome these barriers and access unacknowledged and unrealized needs, UCD often utilizes unconventional activities that elicit tacit and latent knowledge of the participants by observing what participants do, say, or make.

\subsection{Study Population and Recruitment}

Stakeholders were convened into four advisory boards: women, and their support persons, with a history of a periviable delivery (22/0-24/6 weeks) whose children survived (PAB 1) or did not survive as a result of unsuccessful resuscitative efforts or complications in the neonatal intensive care unit (NICU), or because parents' opted for palliation (PAB $2)$; pregnant women within the gestational window of periviability (20/0-25/6 weeks) and their support persons (PAB $3)$; and obstetric and neonatology physicians and nurses (PAB 4). The study schema and UCD strategy are depicted in Fig. 1. We defined a 'support person' as a partner or family member who was or would be directly involved in deliveryor treatment-related decisions for the mother and her baby. To participate in this study, individuals were required to be at least 18 years of age and speak English or Spanish.

Women with a history of a periviable delivery (22/0-24/6 weeks gestation) (PABs 1 and 2) were identified using institutional databases from IU and UCSF of NICU admissions and bereavement nursing records. Using a call script, trained research assistants contacted eligible candidates whose delivery date occurred more than 1 year prior, but within 5 years of the study period. Pregnant women between 20/0 and 25/6 weeks gestation were recruited from outpatient obstetric clinics affiliated with IU and UCSF. These women were encouraged to bring their partner or support person to the session if they so desired. Due to the volume of staff, scheduling constraints, and concerns about power differentials, three separate provider sessions (PAB 4) were conducted: one for physicians and two for nurses. Obstetricians and neonatologists from IU participated in a session conducted over lunch during time slated for their regularly scheduled mother-baby conference. Research assistants conducted two sessions with nurses during weekly staff meetings. Subsequently, research assistants followed up, via email and phone calls, with PAB representatives who expressed an interest in participating in the final session to test prototypes.

\subsection{The 'Explore' Sessions}

Across the two sites, 14 sessions were held with PABs to explore the optimal mode and appropriate timing for delivering an SDM tool in the setting of threatened periviable delivery $(I U=6, U C S F=8)$. Each session began with an icebreaker and warm-up activity to build rapport among the participants and research team members, which was followed by an introduction to the study objectives to frame the session. PAB sessions involved small and large group activities facilitated by the design research team and research assistants. Separate PAB sessions were conducted across the two sites-IU and UCSF-in an effort to capture a more racial/ethnically and regionally diverse population. In particular, two UCSF panels were conducted in Spanish to enrich our sample with Hispanic/Latina participants. One or more of the following design research activities were utilized to elicit a large amount of participant-generated data.

\subsubsection{Experience Mapping (Periviable PABs 1 and 2)}

Experience mapping is an exploratory activity in which participants are asked to draw out their movements and interactions during a particular experience (e.g., periviable delivery) [29]. This can illuminate the holistic highs and lows people experience during a particular interaction and may uncover key moments that, once improved, will unlock a more valuable overall experience. Participants in the periviable PAB sessions were given a large sheet of paper with an $\mathrm{S}$-shaped timeline with three bullet prompts to guide an indepth discussion: (1) the time you learned that you were at risk of having an early delivery, (2) the moment in the delivery room, and (3) your life 1-year postpartum (Fig. 2). The facilitator further prompted participants by asking them to imagine themselves in that moment and describe the setting, the people involved, and their reactions in the moment. A design research specialist captured each person's experience on a sticky note and added it to the paper timeline, creating a large map of shared experiences.

\subsubsection{Icon Collage (Pregnant PAB 3)}

Icon collage is a visualizing technique where participants are asked to express themselves in media other than words. This type of approach aids in common understanding, allows ideas to be shared and discussed, and reveals relationships that might not be accessible in verbal presentations [30]. Pregnant women and their partners were asked to imagine being in a hypothetical scenario in which they learned that they were at risk of delivering very early and had to decide whether to pursue life-supporting measures or comfort care. Participants were given a set of 20, one-inch round icons of objects that are not directly related to periviable delivery 

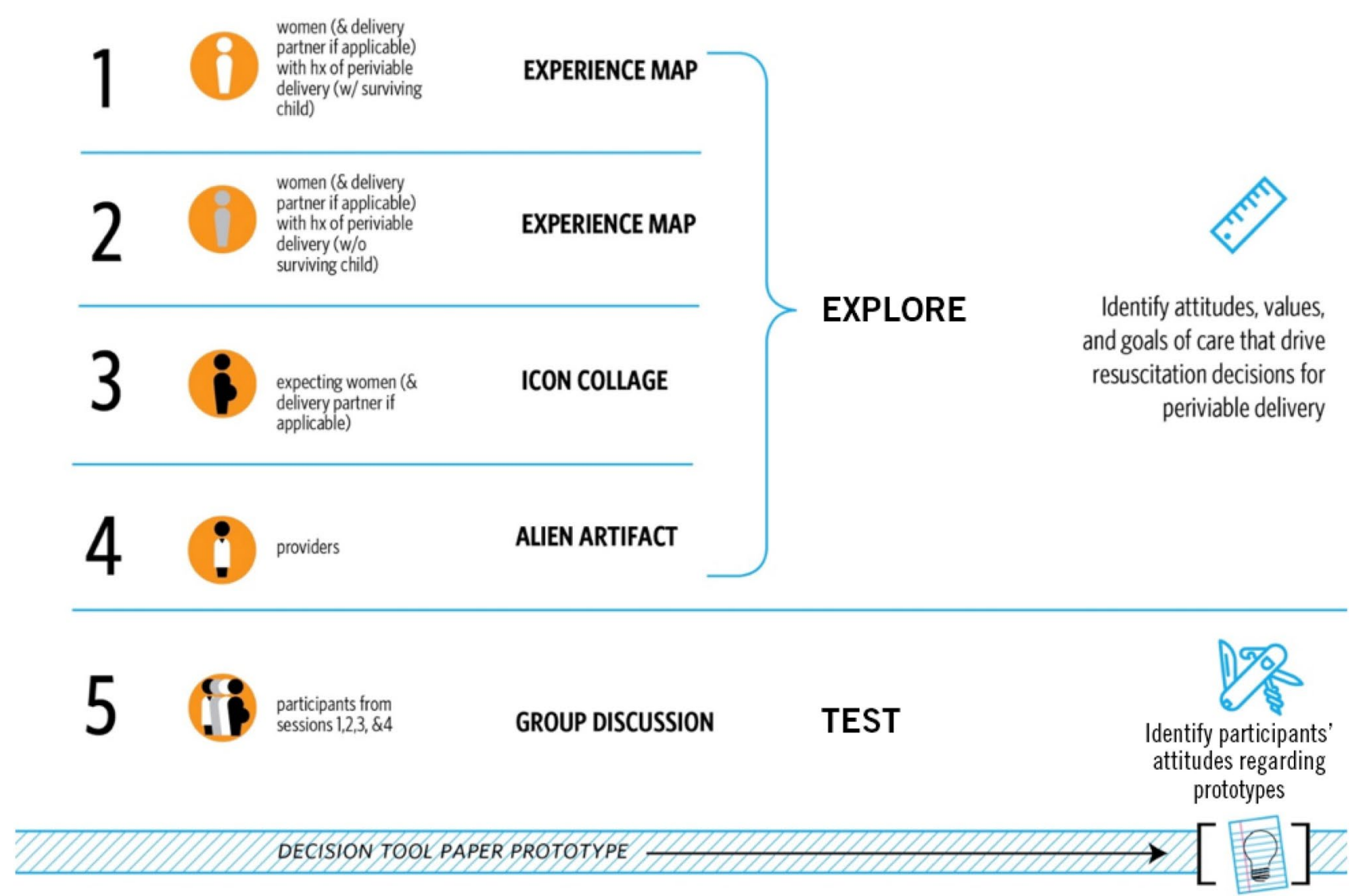

Fig. 1 Study schema for sessions $1-5$. The figure illustrates the overall study plan to explore and test sessions, specifically noting the target population for each session and the research design methodology

(e.g., book, dollar sign, bed), people figures, body parts (i.e., eye, ear) and colors and one large, white sheet of paper with a box drawn on it that represented the decision for resuscitation or palliation. Participants were instructed to create a collage by (1) gluing icons inside the parameters of the box representing the items, thoughts, or feelings that they would utilize in making a treatment decision for their baby, and (2) gluing icons outside of the box that they would definitely not want during the decision-making process. All neutral icons were left off the paper. See Fig. 3 for an example of a completed activity. Finally, participants were asked to show their collage to the group and explain aloud why they chose the images they chose.

\subsubsection{Alien Artifact (Provider PAB 4)}

Alien Artifact is a generative drawing activity that encourages participants to envision 'blue sky' concepts for solving problems related to the topic of discussion. Participants are asked to imagine that aliens from another planet invented a device that helps them solve a problem that would be incorporated into each session activity. $h x$ history, $w /$ with, $w / o$ without

or accomplish a certain outcome related to the topic [28]. They are then prompted to imagine and draw the device on a worksheet, and then describe their device in a group discussion. Physicians and nurses were asked to imagine an artifact that would help them support patients when making general difficult decisions. After a brief round table discussion, researchers asked the providers to draw another alien artifact that would help them support mothers and their families facing the threat of a periviable delivery. Another round of discussion ensued. See Fig. 4a, b for examples.

\subsubsection{Analysis of the Initial PAB Sessions}

Transcripts, observer notes, and activity sheets were reviewed and analyzed by the Patient Engagement Core. Data from all four sessions were transcribed as concepts onto sticky notes, which are traditionally used in peoplecentered design research [31], and then combined into key themes that were arranged into a matrix of the 'should haves', 'could haves', and 'can't haves' of the tool [32]. 


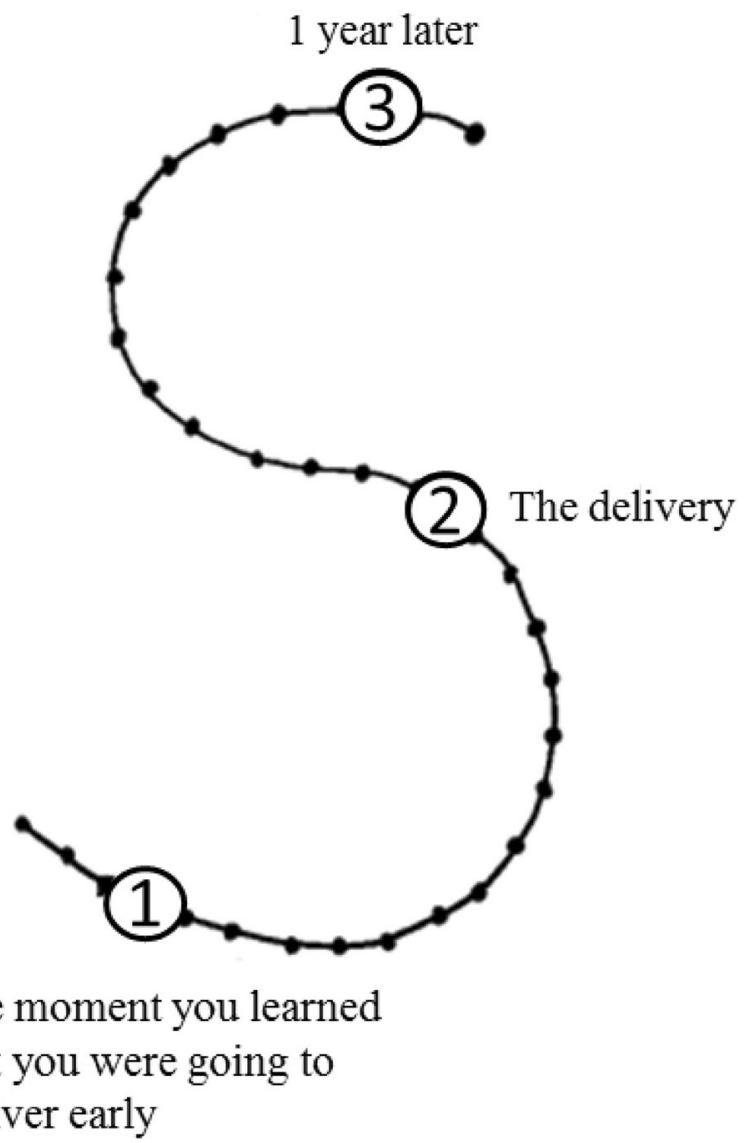

Fig. 2 Experience mapping activity. In this experience mapping activity, women who experienced a periviable delivery and their support partners were encouraged to discuss key events throughout their pregnancy and delivery experiences using a S-shaped timeline to specifically prompt discussion of three specific events: (1) the moment they learned that they were at risk of having an early delivery, (2) the delivery room experience, and (3) their life 1-year postpartum
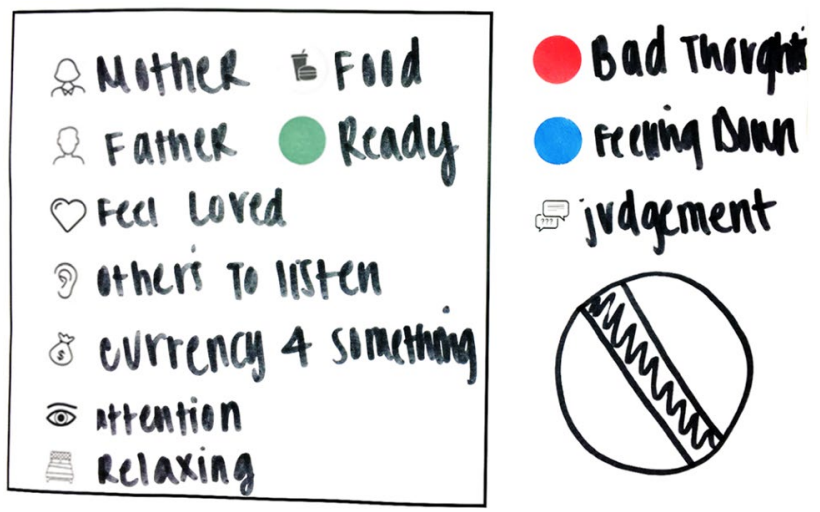

Fig. 3 Icon collage activity sheet example. Shown is an example of the icon collage activity, which prompted pregnant women and their partners to identify items, thoughts, or feelings (represented by icons) that they would want or not want when making treatment decisions in the event of a hypothetical periviable delivery

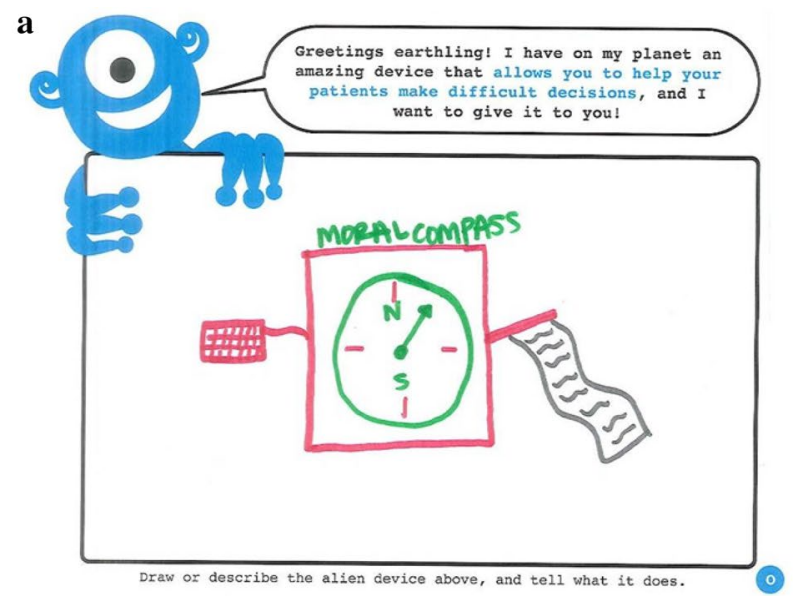

b

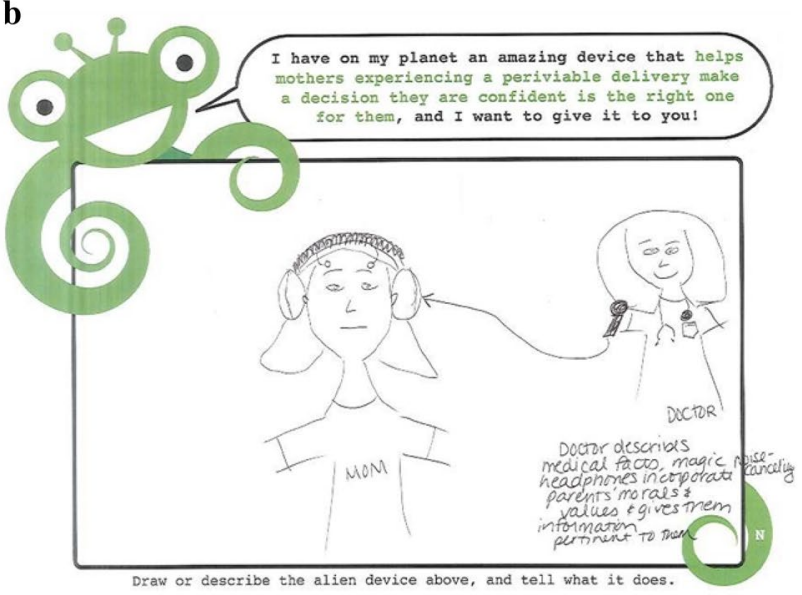

Fig. 4 a Example of an Alien Artifact tool for general difficult decision-making. Shown is an example of one provider's imaginary device that would help them support their patients who are faced with making difficult general decisions regarding their healthcare. In this example, a provider created a "Moral Compass Machine". "You put in about the patient and you can kind of know where their values lie. You can know how to counsel that patient based on what those are". b Example of an Alien Artifact tool for periviable delivery decisionmaking. Shown is an example of a provider's drawing of a device that would help them support mothers and their families specifically facing the threat of a periviable delivery. In this example, a provider drew a set of magical noise-cancelling headphones that are connected to a microphone. "The [doctor] goes through all the medical facts about what's going on. And the magic headphones, these two little electrodes, sense what they're beliefs are and then just interprets the information and tells them basically what their beliefs and morals are"

Using this matrix, the design researchers then engaged in brainstorming sessions to translate stakeholder insights. Unrefined, 'wild ideas' are deconstructed into refined concepts that are then narrowed into prototypes that can be applied in the real-world setting [33]. Specifically, designers seek to use these insights to inspire prototypes that will be feasible, effective, and desirable for the stakeholders. Three GOALS DST prototypes were generated through 
analysis of the stakeholder sessions: a tablet application (app), family story videos, and a virtual reality experience.

\subsection{The'Test' Session}

The team presented the three GOALS DST prototypes at the final session with a subset of representatives for each of the PABs to obtain feedback on the acceptability of each proposed delivery platform. At the start of the session, participants were divided into three groups, with at least one PAB representative in each group. A prototype was set up at each station with a facilitator to demonstrate and ask follow-up questions for feedback. After all participants had interacted with each prototype, a group discussion was held to discuss the participants' thoughts, suggestions, and concerns.

\subsubsection{Prototype 1: Tablet Application}

The first prototype was presented in paper format with a facilitator explaining step by step how it would ideally work if programmed into a tablet app (see the electronic supplementary material, Online Resource 1). The likelihood of several outcomes, including neonatal death and survival with mild, moderate, or severe disabilities, was displayed, and users could click on a link to learn more about each outcome. Additionally, the tablet app included values clarification questions to help the patient think through her primary concerns and anxieties related to the decision she was facing. The final screen provided a print out of the estimated neonatal outcomes and values clarification responses to be shared with providers to facilitate further discussion regarding the best course of action.

\subsubsection{Prototype 2: Family Story Videos}

The second prototype consisted of a storyboard for a short documentary-style video describing the day-to-day lives of children with various outcomes resulting from extremely preterm birth. Online Resource 2 (see the electronic supplementary material) illustrates just one example of neonatal outcomes that may result from delivery at 23 weeks gestation. Participants were informed that these outcomes vary based on additional factors such as gestational age, antenatal steroid use, birth weight, etc. A facilitator presented the storyboard in paper format and explained that its purpose was to help families visualize terms such as 'moderate physical and mental disabilities' that providers use when discussing potential outcomes of periviable deliveries. The storyboard included three stories about Corey, Bobbie, and Anna to illustrate, mild, moderate, and severe disabilities, respectively.

\subsubsection{Prototype 3: Virtual Reality Experience}

The third prototype used virtual reality goggles, an iPhone, and a YouTube video to generate a periviable delivery room virtual reality experience, as videos pertaining to resuscitation and the NICU experience are not readily available. A facilitator explained the scenario for this DST, in which a virtual reality physician introduces three different scenarios: resuscitation, the NICU experience, and a day in the life of a family 1-2 years after experiencing a periviable birth (see the electronic supplementary material, Online Resource 3). Because these scenarios were not available in video format, a facilitator first explained the scenario to the participants, and then, to get a greater understanding of how virtual reality works, participants watched a video documenting a delivery in the hospital, using the goggles.

\subsubsection{Analysis}

Recommendations for the final prototype version were based on design research analysis and incorporated feedback from PABs in the final session. Session audio and video recordings were reviewed and session materials were analyzed using a blend of design research and qualitative descriptive methodologies similar to analysis of the initial PAB sessions, including Ackoff's Data-Information-Knowledge-Wisdom scheme [34]. Key themes were transcribed onto Post-it Notes [31, 32]. Using a criteria grid, the design research team individually assessed the feasibility, desirability, and effectiveness of all three prototypes based on stakeholder feedback to recommend a final prototype.

\section{Results}

Ninety-five stakeholders participated in the preliminary $\mathrm{PAB}$ sessions at IU and UCSF (48 mothers/support persons; 47 providers) between August and December 2016. Stakeholder participant demographic characteristics are presented in Table 1.

\subsection{The 'Explore' Sessions}

Key insights and themes from the preliminary PAB sessions were analyzed to formulate the 'should haves', 'could haves', and 'cannot haves' of the DST (Table 2). Both parents and providers agreed that the tool should be factual and present statistical outcomes. Parents wanted a tool that included comprehensive treatment options and subsequent outcomes, regardless of whether those outcomes are positive or negative. They emphasized that the language should be neutral, 
Table 1 Participant demographics

\begin{tabular}{lrrr}
\hline & UCSF & IU & Total \\
\hline Role & 6 & 10 & 16 \\
Periviable mom & 0 & 5 & 5 \\
Periviable partner & 17 & 5 & 22 \\
Pregnant women & 0 & 5 & 5 \\
Partner of pregnant women & 0 & 17 & 17 \\
Physicians & 0 & 30 & 30 \\
Nurses & 23 & 72 & 95 \\
Total & & & \\
Gender & 23 & 62 & 85 \\
Female & 0 & 10 & 10 \\
Male & & & \\
Race/ethnicity & 0 & 28 & 28 \\
African American or black & 3 & 2 & 5 \\
Asian or Pacific Islander & 12 & 37 & 49 \\
Caucasian, white or European American & 6 & 5 & 11 \\
Latina, Latin American, or Hispanic & 1 & 0 & 1 \\
Mixed & 1 & 0 & 1 \\
Other & & & \\
\hline
\end{tabular}

$I U$ Indiana University, UCSF University of California at San Francisco

sensitive, and inclusive, to avoid presenting biased information and excluding partners or support persons. Some families hoped that the tool would address patient values, such as faith and quality of life, as well as explain their legal rights and limitations for delivery and treatment options. Parents also suggested that the tool should be used to prompt a private conversation with providers, and that the providers should take the time to answer any questions that come up for the parents who used the tool. They also felt that providers need to engage with the individual(s) who are directly involved in making delivery- and treatment-related decisions, such as a partner or support person, so that mothers do not feel alone in the decision-making process, and then end on a hopeful note, so that families feel supported by their providers, regardless of the final decisions that they make.

While parents expressed the importance of having statistics and information to help prepare for a possible periviable delivery and minimize uncertainty, providers wanted the DST to be simple, help ease communication with their patients, and prevent families from experiencing information overload or false hope. Additionally, they suggested incorporating testimonials with statistics to help families visualize and comprehend the potential outcomes of a periviable delivery, including what it means for children to have mild, moderate, and severe disabilities and what type of care they would require throughout their lifespan. Parental insights helped the design team produce three separate prototypes, each meant to address patient-centered values and functional needs within the context of the experiences families face during this decision.

\subsection{The 'Test' Session}

A subset of 17 PAB representatives attended the final session in January 2017 to evaluate the three prototypes. Overall, the majority of participants responded positively to the user-friendly tablet prototype and found that it provided sufficient and unbiased information that would "calm" and prepare parents before engaging in conversations with their providers. They also provided suggestions to help improve aspects of the prototype, such as having a member of the medical staff introduce the tablet app to families to make it feel more personal. They suggested that presenting the statistical outcomes as graphs and charts would illustrate the data for individuals who may not comprehend percentages. The purpose of the values clarification activities was unclear to some participants. As such, they suggested adding language to explain how eliciting values will help patients make delivery and treatment decisions that reflect what is most important to them and to note that any response will not affect their care.

The family story videos prototype also received positive feedback, as participants believed that it provided a realistic, yet "warm" and "hopeful" visualization of the outcomes that can result from a periviable delivery. The videos created a common language that both providers and families understood by providing real-life stories that depicted the components of mild, moderate, and severe disabilities. Some participants suggested including additional videos of palliation and resuscitation in a manner that is informative but not traumatizing. They also wanted the videos to discuss the long-term outcomes that go beyond childhood and address how these outcomes may affect families, including finances and the support required to care for a disabled child. Participants stressed the importance of communicating to families that these stories are only examples and that the experiences of one family should not be compared to another.

The virtual reality experience was not widely accepted among our participants, as many were concerned that viewing newborn resuscitation may be traumatizing to families. Additionally, they were concerned that the technology would not be user friendly. Participants were also concerned about the feasibility of filming certain scenarios, such as palliative care, in a sensitive manner. Since virtual reality is a relatively new technology, they also felt that some families may feel uncomfortable using it due to lack of experience and knowledge of how to work the equipment. Furthermore, the prototype is limited to one user at a time, preventing mothers and their partners or support persons from engaging in conversation while 
Table 2 Preliminary PAB session findings

\begin{tabular}{|c|c|c|c|}
\hline & Should have & Might have & Cannot have \\
\hline \multirow[t]{8}{*}{$\begin{array}{l}\text { Moms and support } \\
\text { persons (PABs 1-3) }\end{array}$} & $\begin{array}{l}\text { Talk about all outcomes, not just good/ } \\
\text { bad }\end{array}$ & Include partner/support person & Exclude partner/support person \\
\hline & Comprehensively explain options & Be an early warning system & Have a forceful demeanor \\
\hline & Have empathy (listening for cues) & Include $1: 1$ conversation with doctors & Be cold/unfeeling \\
\hline & Be factual & Account for faith & Be biased \\
\hline & $\begin{array}{l}\text { Provide a holistic picture (mother's } \\
\text { health/impact on family) }\end{array}$ & Answer "why did this happen?" & Be one-sided (exclude mom's input) \\
\hline & $\begin{array}{l}\text { Discuss quality vs. quantity of baby's } \\
\text { life }\end{array}$ & & $\begin{array}{l}\text { Make assumptions (e.g., you have } \\
\text { time for another baby, etc.) }\end{array}$ \\
\hline & Discuss rights, options, and limitations & & \\
\hline & Normalize long-term decisions & & \\
\hline \multirow[t]{6}{*}{ Care providers (PAB 4) } & Be simple and intuitive & Be immersive & Overwhelm with options \\
\hline & Show probabilities & Be self-guided & Be a bunch of handouts \\
\hline & $\begin{array}{l}\text { Ease communication between provider } \\
\text { and patient }\end{array}$ & $\begin{array}{l}\text { Provide access to other similar patient } \\
\text { testimonials/ experiences }\end{array}$ & \\
\hline & Reveal patient's values & $\begin{array}{l}\text { Lessen some responsibility from the } \\
\text { mom }\end{array}$ & \\
\hline & Combine stories and statistics & & \\
\hline & Include partner/support person & & \\
\hline
\end{tabular}

$P A B$ patient and provider advisory board

using the tool. As such, if the first user became upset while watching the video, the next individual would have to wait to know what was upsetting, rather than seeing it in the moment.

\subsection{Final Prototype Design}

Based on findings from the final test session, it was decided that the tablet app was the most feasible, effective, and desirable tool for GOALS that both mothers and their partners or support persons can use together, with family story videos to supplement neutral and unbiased information with real stories. The developers agreed that the tool should be used to facilitate and not replace patient-provider conversation and provide information that is sensitive and realistic, but still hopeful. We also conclude that the app should incorporate graphics (e.g., pie charts, icon arrays) that update in real time based on user input and that should include links for users to have access to more information about outcomes. Based on additional recommendations from the co-design process, the family story videos will encompass a range of outcomes, including palliation, and the DST will communicate to users that these stories are only a few examples along a spectrum of possibilities. The values clarification exercises will also address social concerns, such as finances and family support systems. Ultimately, a summary report of individualized outcome estimations and the patient's responses to the values questions will be displayed at the end of the app so that they can be printed and discussed with the care team. This summary report should be used to facilitate effective SDM so that providers can clearly communicate treatment options, the risks and benefits of each option and the alternatives, and the uncertainty that surrounds each option, while also creating a window of opportunity for patients to communicate their values surrounding death and disability that may drive their decision-making for resuscitation or palliation.

\section{Discussion}

In an effort to improve the current quality of periviable counseling and to facilitate more patient- and family-centered periviable care, we set out to engage patients and providers in a user-centered research and co-design process to develop a DST prototype that will help expectant parents facing threatened periviable delivery engage in informed SDM regarding whether to opt for comfort care or resuscitation. After completing this process, we concluded that a DST that combines unbiased outcome estimates and values clarifications alongside family story videos would be most meaningful for patients and providers engaging in SDM in this context.

We utilized a novel application of UCD methodology to ensure that patients, family members, and providers were true partners in every stage of design, and we anticipate 
continuing to engage these partners in future, testing and ultimately disseminating the tool. Clinical experts have stressed the importance of assessing individualized goals of care rather than following traditional clinical guidelines prescribed by gestational age [5]. The decision to resuscitate or palliate is highly 'preference sensitive', as the 'right answer' for a given woman and her family will depend largely on their values and perceptions related to death and disability, and contextual factors, such as support systems and other coping resources. Unfortunately, little is currently known about parents' goals of care-particularly their attitudes and perceptions related to the death or long-term disability that may result from periviable birth. Rather than relying solely on researcher or provider opinion to fill in these gaps, we used data obtained from our PABs to understand the perspective of pregnant women facing the threat of periviable delivery.

Concerns about the ethical implications of the use of DSTs have recently been raised, focusing on their potential to have powerful effects on patients' decision-making. Chief among these concerns is the possible introduction of developers' biases into the tools themselves [35]. UCD is a critically important method that can mitigate the potential for this type of bias and potential for ethical harm, as it fosters collaboration between design researchers and end users to leverage the expertise of all parties [36] by using an iterative process that utilizes participatory methods to draw out the 'native expertise' of stakeholders [37]. Our PABs included women who had a periviable delivery in the past and their family members and women who were pregnant and their family members. Employing this patient-informed refinement process not only mitigates the role of researcher bias, but also allows for improved effectiveness, usability, uptake and dissemination.

Our study is not without limitations. Because the PAB members were recruited from two academic tertiary care centers, their perspectives may not be representative of patients and providers in community-based health systems. However, academic health systems provide the bulk of high-risk obstetrical and neonatal intensive care, making this an important population of focus. The geographic, racial, and ethnic diversity gained from our multi-site study ultimately strengthens the generalizability of our findings to other academic centers across the nation. Further strengths of the work include the inclusion of important others/support persons, allowing us to gain broader perspectives in support of promoting familycentered care. However, our findings are limited because a relatively small portion of women participating in the study presented with a partner. Given these small numbers, additional larger studies are needed to gain adequate understanding of partner perspectives.

Despite these limitations, this work fills a critical gap in decision support design and intervention implementation for pregnant women and their families facing periviable delivery.
Guillen et al. published a report of a card-based decision aid to promote more informed periviable decision-making [38] and later paired it with video [39]. Moore et al. subsequently adapted that decision aid for use with a decision coaching model [40]. These authors utilized focus groups to engage stakeholders in developing and reviewing the content of their tools and, in doing so, made novel contributions in the arena of periviable decision support. However, to our knowledge, there currently are no published studies of tools that have explicitly incorporated values clarification exercises into the decision support process. Furthermore, this new tool is interactive and programmable (i.e., can be reprogrammed as outcomes change or improve over time) to enable patients to obtain personalized estimates of neonatal survival and neurodevelopmental impairment. Lastly, our UCD methodology incorporated extensive and on-going input from nearly 100 stakeholders, including parents and family members who had experienced periviable birth, naïve pregnant women, obstetricians, neonatologists and nurses, providing important insights and perspectives. These represent important advancements in both our tool development approach and the decision support product. These advancements offer the promise of substantial improvements in the quality and patient-centeredness of periviable counseling and care.

\section{Conclusion}

In closing, our results suggest that a DST that combines unbiased information and understandable outcomes with family testimonials can make a meaningful contribution to SDM in periviable care. We found UCD to be a useful method for creating an app-based DST prototype. By leveraging the expertise of a wide range of stakeholders, we expect that this method will lead to improved effectiveness, usability, uptake, and dissemination of the tool in the future.

Author contributions BTE and MK contributed to study concept and design and writing of the manuscript. SMH and EJ participated in subject recruitment, conducting sessions, and writing the manuscript. DL and KJ developed the session content and participated in conducting sessions, analyzing data, and writing the manuscript. SW and NB managed the session content and conduct of sessions, and participated in writing the manuscript.

Data availability The datasets generated during and/or analyzed during the current study are available from the corresponding author on reasonable request.

\section{Compliance with Ethical Standards}

Conflict of interest Authors, BTE, SMH, DL, EJ, KJ, SW, NB, and MK, declare that they have no conflict of interest.

Funding This project was funded, in part, with support from the Indiana Clinical and Translational Sciences Institute and, in part, by 
Award Number UL1TR002529 from the National Institutes of Health, National Center for Advancing Translational Sciences, Clinical and Translational Sciences Award. The content is solely the responsibility of the authors and does not necessarily represent the official views of the National Institutes of Health. This research was supported by the University of California, San Francisco, California Preterm Birth Initiative, funded by Marc and Lynne Benioff.

Research involving human participants This study was approved to include human subjects by the Indiana University Institutional Review Board (IRB protocol \#1606279321) and the University of California, San Francisco Institutional Review Board (IRB protocol \#16-20705).

Ethical approval All procedures performed in studies involving human participants were in accordance with the ethical standards of the institutional and/or national research committee and with the 1964 Helsinki declaration and its later amendments or comparable ethical standards. This article does not contain any studies with animals performed by any of the authors.

Informed consent Informed consent was obtained from all individual participants included in the study.

\section{References}

1. American College of Obstetricians and Gynecologists. Periviable birth. Obstetric Care Consensus No. 6. Obstet Gynecol. 2017;130:e187-99.

2. Kaempf JW, Tomlinson MW, Campbell B, Ferguson L, Stewart VT. Counseling pregnant women who may deliver extremely premature infants: medical care guidelines, family choices, and neonatal outcomes. Pediatrics. 2009;123(6):1509-15.

3. Carlo WA, McDonald SA, Fanaroff AA, et al. Association of antenatal corticosteroids with mortality and neurodevelopmental outcomes among infants born at 22 to 25 weeks' gestation. JAMA. 2011;306(21):2348-58.

4. Tyson JE, Parikh NA, Langer J, Green C, Higgins RD. Intensive care for extreme prematurity-moving beyond gestational age. $\mathrm{N}$ Engl J Med. 2008;358(16):1672-81.

5. Moore GP, Lemyre B, Barrowman N, Daboval T. Neurodevelopmental outcomes at 4 to 8 years of children born at 22 to 25 weeks' gestational age: a meta-analysis. JAMA Pediatr. 2013;167(10):967-74.

6. Hintz SR, Kendrick DE, Wilson-Costello DE, et al. Earlychildhood neurodevelopmental outcomes are not improving for infants born at $<25$ weeks' gestational age. Pediatrics. 2010;127(1):62-70.

7. Younge N, Goldstein RF, Bann CM, et al. Survival and neurodevelopmental outcomes among periviable infants. N Engl J Med. 2017;376(7):617-28.

8. Petrou S, Mehta Z, Hockley C, Cook-Mozaffari P, Henderson J, Goldacre M. The impact of preterm birth on hospital inpatient admissions and costs during the first 5 years of life. Pediatrics. 2003;112(6 Pt 1):1290-7.

9. Clements KM, Barfield WD, Ayadi MF, Wilber N. Preterm birthassociated cost of early intervention services: an analysis by gestational age. Pediatrics. 2007;119(4):e866-74.

10. Gilbert WM, Nesbitt TS, Danielsen B. The cost of prematurity: quantification by gestational age and birth weight. Obstet Gynecol. 2003;102(3):488-92.

11. Institute of Medicine (US) Committee on Understanding Premature Birth and Assuring Healthy Outcomes. In: Behrman RE, Butler AS, editors. Preterm birth: causes, consequences, and prevention. Washington (DC): National Academies Press (US); 2007.

12. Youngblut JM, Brooten D, Cantwell GP, del Moral T, Totapally B. Parent health and functioning 13 months after infant or child NICU/PICU death. Pediatrics. 2013;132(5):e1295-301.

13. Batton DG. Clinical report-antenatal counseling regarding resuscitation at an extremely low gestational age. Pediatrics. 2009; 124(1):422-7.

14. Cummings J. Antenatal counseling regarding resuscitation and intensive care before 25 weeks of gestation. Pediatrics. 2015;136(3):588-95.

15. Boss RD, Hutton N, Sulpar LJ, West AM, Donohue PK. Values parents apply to decision-making regarding delivery room resuscitation for high-risk newborns. Pediatrics. 2008;122(3):583-9.

16. Raju TN, Mercer BM, Burchfield DJ, Joseph GF. Periviable birth: executive summary of a Joint Workshop by the Eunice Kennedy Shriver National Institute of Child Health and Human Development, Society for Maternal-Fetal Medicine, American Academy of Pediatrics, and American College of Obstetricians and Gynecologists. J Perinatol. 2014;34(5):333-42.

17. Kavanaugh K, Savage T, Kilpatrick S, Kimura R, Hershberger P. Life support decisions for extremely premature infants: report of a pilot study. J Pediatr Nurs. 2005;20(5):347-59.

18. Kavanaugh K, Roscigno CI, Swanson KM, Savage TA, Kimura RE, Kilpatrick SJ. Perinatal palliative care: parent perceptions of caring in interactions surrounding counseling for risk of delivering an extremely premature infant. Palliat Support Care. 2015;13(2):145-55.

19. Roscigno CI, Savage TA, Kavanaugh K, et al. Divergent views of hope influencing communications between parents and hospital providers. Qual Health Res. 2012;22(9):1232-46.

20. Tucker Edmonds B, McKenzie F, Panoch JE, Frankel RM. Comparing neonatal morbidity and mortality estimates across specialty in periviable counseling. J Matern Fetal Neonatal Med. 2015;28(18):2145-9.

21. Tucker Edmonds B, McKenzie F, Panoch JE, Barnato AE, Frankel RM. Comparing obstetricians' and neonatologists' approaches to periviable counseling. J Perinatol. 2015;35(5):344-8.

22. Tucker Edmonds B, McKenzie F, Panoch JE, Wocial LD, Barnato AE, Frankel RM. "Doctor, what would you do?": physicians' responses to patient inquiries about periviable delivery. Patient Educ Couns. 2015;98(1):49-54.

23. Tucker Edmonds B, McKenzie F, Panoch JE, White DB, Barnato AE. A pilot study of neonatologists' decision-making roles in delivery room resuscitation counseling for periviable births. AJOB Empir Bioeth. 2016;7(3):175-82.

24. Courage $C$, Baxter $K$. Understanding your users: a practical guide to user requirements methods, tools, and techniques. San Francisco: Elsevier; 2005.

25. Norman DA, Draper SW. User centered system design: new perspectives on human-computer interaction. Hillsdale: Lawrence Erlbaum Associates; 1986.

26. Giacomin J. What is human centred design? Des J. 2014;17(4):606-23.

27. Morgan DL. Focus groups. Annu Rev Sociol. 1996;22:129-52.

28. Sanders EB-N, Brandt E, Binder T. A framework for organizing the tools and techniques of participatory design. In: Proceedings of the participatory design conference 2010, Sydney.

29. Schauer B, Quattlebaum P, Risdon C, Bowman B, Daghlian P, Brune R, et al. Adaptive path's guide to experience mapping. San Francisco and Austin: Adaptive Path; 2013. http://mappingexp eriences.com/. Accessed 15 Nov 2018.

30. Hassi L, Laakso M. Making sense of design thinking. In: Karjalainen TM, Koria M, Salimäki M, editors. IDM papers, vol. 1. Helsinki: IDBM Program, Aalto University; 2011. https:// www.researchgate.net/profile/Mikko_Koria/publication/30129 
3326_IDBM_Papers_Vol1/links/5710370f08aefb6cadaaa721/ IDBM-Papers-Vol1.pdf?origin=publication_detail. Accessed 15 Nov 2018.

31. Gibbons S. In defense of post-its. Nielson Norman Group; 2018. https://www.nngroup.com/articles/post-it-in-ux/. Accessed 5 Nov 2018.

32. Hanington B. Universal methods of design: 100 ways to research complex problems, develop innovative ideas, and design effective solutions. Beverly: Rockport Publishers; 2012.

33. Basadur M. The power of innovation: how to make innovation a way of life and put creative solutions to work. London: Pitman Publishing; 1995.

34. Ackoff RL. From data to wisdom. J Appl Syst Anal. 1989;16:3-9.

35. Guillen U, Kirpalani H. Ethical implications of the use of decision aids for antenatal counseling at the limits of gestational viability. Semin Fetal Neonatal Med. 2018;23(1):25-9.
36. Sanematsu H, Wiehe S. Learning to look: design in health services research. Touchpoint, TP06-02P82; 2014.

37. Sanders E, Stappers P. Co-creation and the new landscapes of design. CoDesign. 2008;4(1):5-18.

38. Guillen U, Suh S, Munson D, et al. Development and pretesting of a decision-aid to use when counseling parents facing imminent extreme premature delivery. J Pediatr. 2012;160(3):382-7.

39. Guillen U, Suh S, Wang E, Stickelman Y, Kirpalani H. Development of a video decision aid to inform parents on potential outcomes of extreme prematurity. J Perinatol. 2016;36(11):939-43.

40. Moore GP, Lemyre B, Daboval T, Ding S, Dunn S, Akiki S, et al. Field testing of decision coaching with a decision aid for parents facing extreme prematurity. J Perinatol. 2017;37(6):728-34. 